Supplement of Nat. Hazards Earth Syst. Sci., 20, 3577-3592, 2020

https://doi.org/10.5194/nhess-20-3577-2020-supplement

(C) Author(s) 2020. This work is distributed under

the Creative Commons Attribution 4.0 License.

(c) (1)

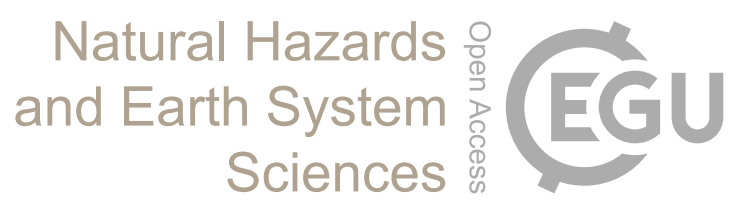

Supplement of

\title{
Style of faulting of expected earthquakes in Italy as an input for seismic hazard modeling
}

Silvia Pondrelli et al.

Correspondence to: Silvia Pondrelli (silvia.pondrelli@ingv.it)

The copyright of individual parts of the supplement might differ from the CC BY 4.0 License. 
Table S1 - Number ( $\mathrm{s}=$ shallow, $\mathrm{d}=$ deep) and names of seismic zones, depth of upper and lower values of thickness attributed to each of them in ZS16 $\left(5^{\circ}\right.$ and $95^{\circ}$ percentile of hypocentral distribution of seismicity with $\mathrm{Mw} \geq 2.5$ ).

\begin{tabular}{|c|c|c|c|}
\hline $\mathrm{N}$. & Seismic Zone & ZS16 depth $5^{\circ}$ pct $(\mathrm{km})$ & ZS16 depth $95^{\circ}$ pct $(\mathrm{km})$ \\
\hline 1 & Idria & 0 & 22 \\
\hline 2 & Slovenia & 0 & 20 \\
\hline 3 & Friuli & 0 & 17 \\
\hline 4 & Valtellina - Alto Adige & 0 & 25 \\
\hline 5 & Innsbruck & 0 & 23 \\
\hline 6 & Grigioni & 0 & 12 \\
\hline 7 & Garda-Soncino & 0 & 21 \\
\hline 8 & Montreux & 0 & 24 \\
\hline 9 & Vallese & 0 & 15 \\
\hline 10 & Western Alps & 4 & 21 \\
\hline 11 & Piemonte & 6 & 32 \\
\hline 12 & Mantova-Verona & 0 & 29 \\
\hline 13 & Pianura veneta & 0 & 25 \\
\hline 14 & Imperiese & 3 & 21 \\
\hline 15 & Mar Ligure & 7 & 21 \\
\hline 16 & Tortona-Bobbio & 1 & 26 \\
\hline 17 & Spezia-North of Tuscany & 1 & 13 \\
\hline 18 & Lunigiana-Casentino & 0 & 17 \\
\hline $19 \mathrm{~s}$ & $\begin{array}{l}\text { Tuscany-Emilia Apennines } \\
\text { Shallow }\end{array}$ & 1 & 15 \\
\hline $19 d$ & Tuscany-Emilia Apennines Deep & 15 & 30 \\
\hline $20 \mathrm{~s}$ & Emilia Shallow & 1 & 15 \\
\hline $20 d$ & Emilia Deep & 15 & 30 \\
\hline 21 & Ferrara Arc & 2 & 16 \\
\hline 22 & Geothermal reg. Tuscany Latium & 0 & 17 \\
\hline 23 & Trasimeno-Southern Latium & 0 & 19 \\
\hline 24 & Umbria-Abruzzo & 0 & 14 \\
\hline $25 \mathrm{~s}$ & Inner part of Marche & 1 & 15 \\
\hline $25 d$ & Inner part of Marche & 15 & 30 \\
\hline 26 & Rimini-Conero-Majella & 0 & 30 \\
\hline
\end{tabular}




\begin{tabular}{|c|c|c|c|}
\hline N. & Seismic Zone & ZS16 depth $5^{\circ}$ pct $(\mathrm{km})$ & ZS16 depth $95^{\circ}$ pct $(\mathrm{km})$ \\
\hline 27 & Northern Tyrrhenian Coast & 0 & 22 \\
\hline 28 & Colli Albani & 0 & 20 \\
\hline 29 & Chieti-Pescara & 0 & 27 \\
\hline 30 & Central Adriatic Sea & 0 & 32 \\
\hline 31 & Ischia-Vesuvio & 0 & 4 \\
\hline 32 & $\begin{array}{c}\text { Campania part of the Tyrrhenian } \\
\text { coast }\end{array}$ & 0 & 20 \\
\hline 33 & Sannio-Irpinia & 0 & 17 \\
\hline 34 & Gargano & 0 & 27 \\
\hline 35 & Ofanto & 0 & 20 \\
\hline 36 & Potenza-Matera & 0 & 27 \\
\hline 37 & Southern Puglia & 0 & 28 \\
\hline 38 & Otranto channel & 0 & 11 \\
\hline 39 & $\begin{array}{c}\text { Calabrian part of the Tyrrhenian } \\
\text { coast }\end{array}$ & 0 & 22 \\
\hline 40 & Calabrian part of the Ionian coast & 0 & 23 \\
\hline 41 & Ionian Sea & 2 & 33 \\
\hline 42 & Sardegna-Corsica & 1 & 24 \\
\hline 43 & Ustica-Alicudi & 0 & 18 \\
\hline 44 & Eolie-Patti & 0 & 18 \\
\hline 45 & Cefalù & 0 & 20 \\
\hline 46 & Western Sicily & 0 & 35 \\
\hline 47 & Malta-Lampedusa & 4 & 23 \\
\hline 48 & Iblei & 0 & 31 \\
\hline 49 & Etna & 0 & 5 \\
\hline 50 & Southern Tyrrhenian Sea & 0 & 22 \\
\hline
\end{tabular}




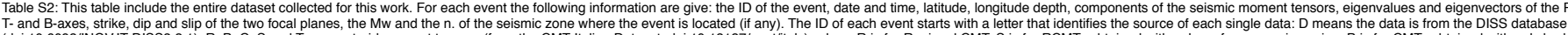

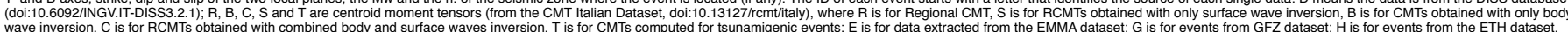

\begin{tabular}{|c|c|c|c|c|c|c|}
\hline ID event & date & time & latitude & longitude & $\begin{array}{c}\text { depth } \\
(\mathrm{km})\end{array}$ & rr \\
\hline E190211280000A & $1902-11-28$ & 00:00:28 & 37.21 & 15.55 & 22.6 & -4.240 \\
\hline D190509080143A & 1905-09-08 & 01:43:00 & 38.80 & 16.19 & 10.0 & -0.970 \\
\hline E190812280420A & $1908-12-28$ & 04:20:28 & 38.10 & 15.40 & 0.0 & -3.400 \\
\hline D191501130652A & 15-01-13 & :52:00 & 41.96 & 3.61 & 10.0 & -0.870 \\
\hline E192803270832A & 1928-03-27 & 08:32:31 & 46.25 & 13.02 & 4.0 & 0.000 \\
\hline E193007230008A & $1930-07-23$ & 00:08:43 & 41.10 & 11.20 & 14.0 & 0.150 \\
\hline E193010080028A & 1930-10-08 & 00:28:48 & 47.33 & 10.78 & 8.0 & 4.090 \\
\hline E193406080313A & 1934-06-08 & 03:13:09 & 46.30 & 12.50 & 20.0 & 0.000 \\
\hline E193610199999A A & $1936-10-19$ & 99:99:00 _ ل & 46.00 & 12.50 & 10.0 & 0.000 \\
\hline E193803279999A A & 1938-03-27 & 99:99:00 & 46.20 & 16.80 & 7.0 & 1.470 \\
\hline E193804130245A & $1938-04-13$ & 02:45:46 & 39.20 & 15.20 & 70.0 & -0.620 \\
\hline E193902111116A & 1939-02-11 & 11:16:00 & 44.07 & 11.03 & 7.0 & -3.180 \\
\hline E193910151505A & 1939-10-15 & 15:05:25 & 44.23 & 10.20 & 26.0 & -0.870 \\
\hline E194103161635A & 1941-03-16 & 16:35:15 & 38.50 & 11.50 & 0.0 & -0.950 \\
\hline E194705110632A & 1947-05-11 & 06:32:00 & 38.69 & 16.78 & 14.0 & -0.720 \\
\hline E195009050409A & 1950-09-05 & 04:09:00 & 42.60 & 13.50 & 10.0 & -3.160 \\
\hline E195105152254A & 1951-05-15 & 22:54:00 & 45.33 & 9.33 & 6.0 & -1.940 \\
\hline E195105160226A & $1951-05-16$ & 02:26:00 & 45.33 & 9.33 & 6.0 & -0.470 \\
\hline E195108082056A & 1951-08-08 & 20:56:00 & 42.60 & 13.50 & 10.0 & 0.820 \\
\hline E195212262355A & $1952-12-26$ & 23:55:00 & 39.96 & 15.55 & 64.0 & -0.410 \\
\hline E195407290442A & 1954-07-29 & 04:42:00 & 46.28 & 7.50 & 10.0 & 0.070 \\
\hline E195411231300A & 1954-11-23 & 13:00:00 & 38.60 & 15.10 & 39.0 & 0.570 \\
\hline E195601120546A & 1956-01-12 & 05:46:08 & 47.50 & 19.30 & 0.0 & 1.180 \\
\hline E195602011510A & 1956-02-01 & 15:10:46 & 39.50 & 16.00 & 0.0 & -1.490 \\
\hline E195608151202A & 1956-08-15 & 12:02:05 & 43.21 & 15.95 & 14.0 & -1.980 \\
\hline E195611051945A & 1956-11-05 & 19:45:25 & 46.34 & 12.58 & 2.0 & 0.000 \\
\hline E195702200440A & 1957-02-20 & 04:40:00 & 36.40 & 9.00 & 0.0 & 0.240 \\
\hline E195705211144A & $1957-05-21$ & 11:44:00 & 38.67 & 14.11 & 5.0 & -0.620 \\
\hline E195804030223A & 1958-04-03 & 02:23:42 & 41.10 & 19.80 & 0.0 & -0.640 \\
\hline E195806240607A & $1958-06-24$ & 06:07:00 & 42.35 & 13.44 & 10.0 & -6.420 \\
\hline E195904051048A & 1959-04-05 & 10:48:00 & 44.53 & 6.78 & 0.0 & -0.670 \\
\hline E195904261445A & 1959-04-26 & 14:45:16 & 46.28 & 13.00 & 5.0 & -0.220 \\
\hline E195908170133A & 1959-08-17 & 01:33:14 & 40.90 & 19.80 & 0.0 & -1.510 \\
\hline E195909011137A & 1959-09-01 & 11::37:40 & 40.90 & 19.80 & 0.0 & 3.640 \\
\hline E195910052034A & 1959-10-05 & 20:34:04 & 40.90 & 19.80 & 0.0 & -1.780 \\
\hline E195910070830A & 1959-10-07 & 08:30:40 & 40.90 & 19.90 & 0.0 & 0.380 \\
\hline E195912230929A & $1959-12-23$ & 09:29:00 & 37.72 & 14.61 & 77.0 & 0.680 \\
\hline E196001032019A & $1960-01-03$ & 20:19:30 & 39.50 & 15.50 & 50.0 & -1.910 \\
\hline E196003232308A & 1960-03-23 & 23:08:00 & 46.35 & 8.07 & 12.0 & 3.340 \\
\hline E196010032019A & 1960-10-03 & 20:19:00 & 39.26 & 15.29 & 83.0 & -2.280 \\
\hline E196104282048A & $1961-04-28$ & 20:48:00 & 47.70 & 7.90 & 0.0 & 0.000 \\
\hline E196106220056A & 1961-06-22 & 00:56:00 & 42.32 & 19.26 & 24.0 & 4.750 \\
\hline E196201071003A & 1962-01-07 & 10:03:01 & 43.20 & 88 & 10.0 & 6.700 \\
\hline E196201110505A & 1962-01-11 & 05:05:00 & 43.15 & 16.94 & 10.0 & 0.550 \\
\hline E196201210251A & 1962-01-21 & 02:51:03 & 43.09 & 16.95 & 14.0 & 0.280 \\
\hline E196203181530A & 1962-03-18 & 15:30:30 & 40.70 & 19.60 & 0.0 & 1.160 \\
\hline E196203252138A & 1962-03-25 & 21:38:00 & 39.12 & 14.55 & 43.0 & -0.260 \\
\hline E196204171003A & $1962-04-17$ & 10:03:04 & 42.15 & 17.07 & 21.0 & -0.160 \\
\hline E196206110715A & 1962-06-11 & 07:15:04 & 43.60 & 18.40 & 8.0 & -1.000 \\
\hline E196208211809A & $1962-08-21$ & 18:09:00 & 41.00 & 15.00 & 0.0 & -2.650 \\
\hline E196208211844A & 1962-08-21 & 18:44:00 & 41.18 & 15.09 & 36.0 & 0.140 \\
\hline E196208211819A & 1962-08-21 & 18:19:33 & 41.40 & 15.50 & 34.0 & -0.940 \\
\hline E196302141318A & 1963-02-14 & 13:18:05 & 44.26 & 15.17 & 15.0 & -2.530 \\
\hline E196305191000A & 1963-05-19 & 10:00:00 & 46.19 & 14.72 & 14.0 & 0.140 \\
\hline E196307190546A & 1963-07-19 & 05:46:04 & 43.34 & 8.04 & 14.0 & 1.100 \\
\hline E196307270557A & 1963-07-27 & 05:57:00 & 43.56 & 8.13 & 14.0 & 0.770 \\
\hline E196309292216A & 1963-09-29 & 22:16:00 & 36.00 & 18.08 & 37.0 & 0.590 \\
\hline E196403140037A & 64-03-14 & 00:37:00 & 46.90 & & 11.0 & 4.750 \\
\hline E196403142044A & 1964-03-14 & 20:44:00 & 46.90 & 8.30 & 0.0 & 0.620 \\
\hline E196404130830A & 1964-04-13 & 08:30:00 & 45.24 & 18.03 & 11.0 & 1.750 \\
\hline E196406301229A & 1964-06-30 & 12:29:59 & 47.36 & 15.48 & 12.0 & 0.210 \\
\hline E196410271946A & 1964-10-27 & 19:46:09 & 47.85 & 15.95 & 1.0 & -0.230 \\
\hline E1965 & 5-10-24 & :16:56 & 46. & 7.48 & 5.0 & -0.860 \\
\hline E196512231529A & $1965-12-23$ & 15:29:00 & 40.53 & 14.87 & 10.0 & -0.850 \\
\hline E196604071958A & 1966-04-07 & 19:58:04 & 44.12 & 7.39 & 6.0 & 1.410 \\
\hline E196605250906A & $1966-05-25$ & 09:06:57 & 40.32 & 19.82 & 21.0 & -0.940 \\
\hline E1966080 & 6-08-05 & 47:04 & 42.10 & 18.80 & 35.0 & -1.1 \\
\hline E196608060231A & $1966-08-06$ & 02:31:03 & 42.18 & 18.79 & 3.0 & -0.550 \\
\hline E196608090334A & $1966-08-09$ & 03:34:15 & 40.22 & 19.86 & 38.0 & -0.250 \\
\hline E196608160353A & $1966-08-16$ & 03:53:41 & 40.16 & 19.75 & 20.0 & 1.860 \\
\hline E196608201908A & 86-08-20 & 19:08:19 & 42.08 & 18.93 & 8.2 & 0.890 \\
\hline E196608201205A & $96-08-20$ & 12:05:19 & 42.18 & 18.65 & 33.0 & 0.580 \\
\hline E196612081131A & 66-12--08 & 11:31:20 & 42.17 & 18.87 & 47.0 & 0.420 \\
\hline E196701290012A & 1967-01-29 & 00:12:12 & 47.53 & 14.19 & 7.0 & -1.030 \\
\hline & 7-05-12 & :53:00 & 44. & 10.67 & 39.0 & -6.280 \\
\hline E196707131438A & 1967-07-13 & 14:38:58 & 40.66 & 19.67 & 73.0 & -0.680 \\
\hline & 77-07-20 & 03:30 & 40.72 & 19.88 & 58.0 & 6.950 \\
\hline E196708150706A & 77-08-15 & 06:00 & 38.71 & 15.28 & 2.0 & 0.860 \\
\hline E196709071409A A & 77-09--07 & :09:00 & 37. & 15.94 & 53.0 & -0.260 \\
\hline E196710312108A & 57-10-31 & 21:08:00 & 37.84 & 14.60 & 38.0 & -1.240 \\
\hline E196712090309A & 1967-12-09 & 03:09:00 & 42.00 & 16.50 & 33.0 & -1.880 \\
\hline & 7-12-30 & 19:00 & $44.6 \mathrm{z}$ & 12.01 & 33.0 & 1.300 \\
\hline E196801150201A & 8-01-15 & 11:08 & 37.75 & 12.98 & 10.0 & 1.310 \\
\hline E196801150133A & $968-01-15$ & :33:00 & 37.80 & 13.00 & 0.0 & 4.060 \\
\hline E196801161642A & 1968-01-16 & 16:42:44 & 37.86 & 12.98 & 36.0 & 2.350 \\
\hline & $8-01-25$ & 56:48 & 37. & 12.97 & 3.6 & 40 \\
\hline & -02-12 & & 37.96 & & 10.0 & 20 \\
\hline & $8-05-19$ & $37: 00$ & 38.52 & 14.82 & 39.0 & 2.440 \\
\hline E196806161303A & $1968-06-16$ & 13:03:00 & 37.78 & 14.65 & 23.0 & 1.370 \\
\hline & $-06-18$ & & & 7. & 12. & \\
\hline E196806221221A & 1968-06-22 & 12:21:37 & 45.81 & & 24.0 & 0.000 \\
\hline
\end{tabular}




\begin{tabular}{|c|c|c|}
\hline ID event & date & time \\
\hline E196808190036A & 1968-08-19 & \begin{tabular}{|l|l|}
$00: 36: 00$ \\
\end{tabular} \\
\hline E196811030449A & 68-11-03 & :49:33 \\
\hline E196901100432A & 969-01-10 & 04:32:03 \\
\hline E196903290143A & 1969-03-29 & 01:43:00 \\
\hline E196904020138A & 69-04-02 & 01:38:00 \\
\hline E196904032212A & 1969-04-03 & 22:12:00 \\
\hline E196900081548A & 1969-04-08 & 15:48:50 \\
\hline E196906012320A & 69-06-01 & 23:20:00 \\
\hline E196908091701A & 1969-08-09 & 17:01:03 \\
\hline E196908091625A & 1969-08-09 & 16:25:35 \\
\hline E196910261536A & $1969-10-26$ & 15:36:04 \\
\hline E196910270810A & $1969-10-27$ & 08:10:05 \\
\hline E197006050920A & 70-06-05 & 09:20:00 \\
\hline E197008180425A & 1970-08-18 & 04:25:31 \\
\hline E197008190201A & 1970-08-19 & 02:01:00 \\
\hline E197008191219A & 1970-08-19 & 12:19:00 \\
\hline E197008250140A & $1970-08-25$ & 01:40:01 \\
\hline E197010311607A & $1970-10-31$ & 16:07:39 \\
\hline E197102011226A & 1971-02-01 & 12:26:06 \\
\hline E197102061809A & 1971-02-06 & 18:09:00 \\
\hline E197102111849A & 1971-02-11 & 18:49:00 \\
\hline E197102120454A & 1971-02-12 & 04:54:00 \\
\hline E197104250439A & $1971-04-25$ & 04:39:00 \\
\hline E197105060345A & 871-05-06 & 03:45:00 \\
\hline E197106062159A & 1971-06-06 & 21:59:11 \\
\hline E197107150133A & 871-07-15 & 01:33:22 \\
\hline E197109251034A & 1-09-25 & 10:34:00 \\
\hline E197109290719A & $971-09-29$ & 07:19:00 \\
\hline E197111291849A & 1971-11-29 & 18:49:00 \\
\hline E197202040242A & 72--02-04 & $02: 4$ \\
\hline E197202040918A & 1972-02-04 & 09:18:00 \\
\hline E197202050126A & 2-02-05 & 01:26:00 \\
\hline E197202060134A & 972-02-06 & 01:34:00 \\
\hline E197203212306A & 72-03-21 & 23:06:00 \\
\hline E197204161010A & 72-04-16 & 10:10:03 \\
\hline E197206141855A & 772-06-14 & 18:55:00 \\
\hline E197209160353A & 2-09-16 & 03:53:26 \\
\hline E197210252156A & $1972-10-25$ & 21:56:13 \\
\hline E197211261603A & $1972-11-26$ & 16:03:00 \\
\hline E197304071930A & 1973-04-07 & 19:30:09 \\
\hline E197304130 & 73-04-13 & 08:12:00 \\
\hline E197306062111A & 1973-06-06 & 21:11:22 \\
\hline E197308081436A & 3-08-08 & 14:36:00 \\
\hline E197308080823A & 1973-08-08 & 08:23:48 \\
\hline E197310300114A & 3-10-30 & $1: 14: 00$ \\
\hline E197312120002A & 1973-12-12 & 00:02:38 \\
\hline E197312201744A & $1973-12-20$ & 17:44:00 \\
\hline E197401241319A & 4-01-24 & 13:19:00 \\
\hline E197404152149A & 1974-04-15 & 21:49:00 \\
\hline E197406202226A & 4-06-20 & 22:26:31 \\
\hline E197406201708A & 4-06-20 & 17:08:28 \\
\hline E197407220719A & 1974-07-22 & 07:19:00 \\
\hline 197410201125A & 4-10-20 & 11:25:50 \\
\hline E197411231846A & 1974-11-23 & 18:46:00 \\
\hline E197412020155A & 774-12-02 & 01:55:00 \\
\hline E197501160009A & 75-01-16 & 00:09:00 \\
\hline E197501241633A & $1975-01-24$ & 16:33:04 \\
\hline E197504121647A & 75-04-12 & 16:47:00 \\
\hline E197505290032A & 5-05-29 & 00:32:00 \\
\hline E197506191011A & 1975-06-19 & 10:11:00 \\
\hline E197508102055A & 5-08-10 & 20:55:00 \\
\hline E197509161845A & 55-09-16 & 18:45:48 \\
\hline E197509160506A & $1975-09-16$ & 05:06:19 \\
\hline & 5-11- & 13:04:00 \\
\hline E197603021941A & 1976-03-02 & 19:41:34 \\
\hline & 6-04-06 & 08:00 \\
\hline E197605061959A & 6-05-06 & 19:59:06 \\
\hline R197605062000A & 76-05-06 & 20:00:12 \\
\hline R197605070023A & 6-05-07 & 00:23:50 \\
\hline R197605090053A & 76-05-09 & 00:53:44 \\
\hline E197605100435A & 876-05-10 & 04:35:52 \\
\hline B197605: & 6-05-11 & 2:44:00 \\
\hline E197606081214A & 1976-06-08 & 12:14:38 \\
\hline B197606171428A & 776-06-17 & 14:28:49 \\
\hline & 6-08-22 & 0 \\
\hline R197609111631A & 1976-09-11 & 16:31:11 \\
\hline $1976 \mathrm{C}$ & 6-09-11 & 35:03 \\
\hline E197609131854A & 6-09-13 & 18:54:46 \\
\hline & 6-09-15 & : \\
\hline E197609150945A & 6-09-15 & :45:57 \\
\hline $9760: 5>$ & 6-09-15 & 21.18 \\
\hline R197609150315A & 76-09-15 & 03:15:18 \\
\hline E197609170123A & 6-09-17 & $: 23: 00$ \\
\hline E197609211501A & 7-09-21 & 15:01:00 \\
\hline & $-10-22$ & \\
\hline E197612130524A & $1976-12-13$ & 05:24:03 \\
\hline & 7-01-13 & 09:19:06 \\
\hline & -03 & \\
\hline B197 & 77-06-05 & 13:59:21 \\
\hline B197706280712A & $977-06-28$ & 07:12:49 \\
\hline & & \\
\hline 2110 & 77-08-15 & 21:10:32 \\
\hline B1977082 & 7-08-28 & \\
\hline & & \\
\hline E197712030539A & 1977-12-03 & 05:39:30 \\
\hline
\end{tabular}




\begin{tabular}{|c|c|c|}
\hline ID event & date & time \\
\hline E197712202004A & 877-12-20 & 20:04:00 \\
\hline E197712301808A & 7-12-30 & :08:00 \\
\hline B197712301735A & 977-12-30 & 17:35:08 \\
\hline B197803111920A & 1978-03-11 & 19:20:49 \\
\hline E197804031049A & 1978-04-03 & 10:49:46 \\
\hline B197804152333A & $1978-04-15$ & 23:33:47 \\
\hline S197807300519A & 1978-07-30 & 05:19:24 \\
\hline E197808131653A & 1978-08-13 & 16:53:00 \\
\hline E197809240807A & 1978-09-24 & 08:07:00 \\
\hline E197812030810A & 1978-12-03 & 08:10:52 \\
\hline S197812051539A & $1978-12-05$ & 15:39:04 \\
\hline E197812121514A & $1978-12-12$ & 15:14:49 \\
\hline B197812271746A & $1978-12-27$ & 17:46:10 \\
\hline T197901201349A & $1979-01-20$ & 13:49:59 \\
\hline E197902091444A & 1979-02-09 & 14:44:00 \\
\hline E197903221932A & 1979-03-22 & 19:32:00 \\
\hline E197903251136A & $1979-03-25$ & 11:36:00 \\
\hline E197904060210A & 1979-04-06 & 02:10:21 \\
\hline B197904090210A & 1979-04-09 & 02:10:20 \\
\hline T197904151025A & 1979-04-15 & 10:25:24 \\
\hline C197904150619A & 1979-04-15 & 06:19:41 \\
\hline E197904151107A & 1979-04-15 & 11:07:30 \\
\hline B197904151443A & 1979-04-15 & 14:43:06 \\
\hline E197904162300A & 1979-04-16 & 23:00:26 \\
\hline E197904161004A & 1979-04-16 & 10:04:39 \\
\hline E197904171806A & 1979-04-17 & 18:06:17 \\
\hline S197904170539A & 1979-04-17 & 05:39:57 \\
\hline E197904180350A & 1979-04-18 & 03:50:06 \\
\hline R197904181519A A & 1979-04-18 & 15:19:19 \\
\hline E197904190017A & 1979-04-19 & 00:17:35 \\
\hline E197904190542A & 1979-04-19 & 05:42:49 \\
\hline E197904210433A & 1979-04-21 & :33:02 \\
\hline E197904220632A & 1979--04-22 & 06:32:13 \\
\hline E197904280338A & 1979--04-28 & 03:38:03 \\
\hline E197904301700A & 1979-04-30 & 17:00:06 \\
\hline S197905120330A & 1979-05-12 & 03:30:34 \\
\hline E197905140953A & 1979-05-14 & 09:53:07 \\
\hline B197905241723A & 1979-05-24 & 17:23:18 \\
\hline E197906202118A & $1979-06-20$ & 21:18:21 \\
\hline B197909192135A & 1979-09-19 & 21:35:37 \\
\hline E197909212347A A & 1979-09-21 & 23:47:00 \\
\hline S197910032257A & $1979-10-03$ & 22:57:52 \\
\hline E197910211131A & 1979-10-21 & 11:31:08 \\
\hline E197911080203A & 1979-11-08 & 02:03:54 \\
\hline B197912080406A & 1979-12-08 & 04:06:34 \\
\hline E198001051432A & $1980-01-05$ & 14:32:29 \\
\hline S198001051432A & 1980-01-05 & 14:32:26 \\
\hline R198002200234A & 1980-02-20 & 02:34:02 \\
\hline E198002200240A & 1980-02-20 & 02:40:00 \\
\hline S198002282104A & $1980-02-28$ & 21:04:40 \\
\hline S198003091203A & 1980-03-09 & 12:03:40 \\
\hline E198003091200A & 1980-03-09 & 12:00:00 \\
\hline R198005140141A & 1980-05-14 & 01:41:03 \\
\hline B198005281951A & 1980-05-28 & 19:51:19 \\
\hline R198006010232A & 1980-06-01 & 02:32:52 \\
\hline S198006071835A & $1980-06-07$ & 18:35:01 \\
\hline S198006091602A & $1980-06-09$ & 16:02:48 \\
\hline S198006142056A & 1980-06-14 & 20:56:50 \\
\hline S198006161444A & $1980-06-16$ & 14:44:34 \\
\hline & 80-06-22 & 14:33 \\
\hline E198007071032A & 1980-07-07 & 10:32:04 \\
\hline E198007151217A & $1980-07-15$ & 12:17:00 \\
\hline E198010102142A & 1980-10-10 & 21:42:52 \\
\hline E198010130344A & $1980-10-13$ & 03:44:00 \\
\hline S198011071108A & $1980-11-07$ & 11:08:40 \\
\hline Z198011231834A & 1980-11-23 & 18:34:53 \\
\hline R198011240023A & $1980-11-24$ & 00:23:59 \\
\hline & 30-11-24 & :03:53 \\
\hline B198011251828A & $1980-11-25$ & 18:28:21 \\
\hline & 80-11-25 & 06:44 \\
\hline E198012020558A & 1980-12-02 & 05:58:00 \\
\hline S198012032354A & $1980-12-03$ & 23:54:24 \\
\hline R198012090550A & $1980-12-09$ & 05:50:13 \\
\hline $\mathrm{S} 198012231201 \mathrm{~A}$ & 1980-12-23 & 12:01:03 \\
\hline & 1981-01-16 & $: 37: 46$ \\
\hline E198102080430A & 1981-02-08 & 04:30:10 \\
\hline S198102141727A & $1981-02-14$ & 17:27:44 \\
\hline E198103261739A & 1981-03-26 & 17:39:45 \\
\hline S198104201260 & 1981-04-22 & 04 \\
\hline E198105100244A & $1981-05-10$ & 02:44:00 \\
\hline & 31-06-07 & $: 00: 5$ \\
\hline R198106220936A & $1981-06-22$ & 09:36:18 \\
\hline 0359A A & 1981-07-08 & :59:52 \\
\hline 30258A & 1-08-13 & \\
\hline & $1981-09-26$ & :54:46 \\
\hline R198111290506A & 1981-11-29 & 05:06:47 \\
\hline S198203 & 1982-03-16 & $50 \cdot 2$ \\
\hline S198203210944A & 1982-03-21 & 09:44:00 \\
\hline & & \\
\hline E198205011600A & 1982-05-01 & 16:00:27 \\
\hline E198207261507A & 1982-07-26 & 15:07:00 \\
\hline & & \\
\hline R198208151509A & $1982-08-15$ & 15:09:49 \\
\hline & 2-10-17 & 45:37 \\
\hline S198210171056A & 882-10-17 & 56:47 \\
\hline
\end{tabular}




\begin{tabular}{|c|c|c|}
\hline ID event & date & time \\
\hline S198210181529A & 1982-10-18 & 15:29:23 \\
\hline E198210290817A & 32-10-29 & 08:17:35 \\
\hline B198211162341A & $1982-11-16$ & 23:41:20 \\
\hline E198211170037A & 1982-11-17 & 00:37:54 \\
\hline E198301050403A & 1983-01-05 & 04:03:29 \\
\hline E198307312052A & 1983-07-31 & 20:52:56 \\
\hline S198308121936A & $1983-08-12$ & 19:36:30 \\
\hline B198311091629A & 1983-11-09 & 16:29:51 \\
\hline E198312250256A & 1983-12-25 & 02:56:10 \\
\hline E198403111155A & 1984-03-11 & 11:55:03 \\
\hline B198403210112A & 1984-03-21 & 01:12:43 \\
\hline E198404151057A & 1984-04-15 & 10:57:53 \\
\hline S198404221739A & $1984-04-22$ & 17:39:23 \\
\hline B198404290502A & 1984-04-29 & 05:02:59 \\
\hline E198405030856A & 1984-05-03 & 08:56:52 \\
\hline B198405071749A & 1984-05-07 & 17:49:41 \\
\hline S198405111314A & 1984-05-11 & 13:14:56 \\
\hline B198405111041A & 1984-05-11 & \begin{tabular}{|l|}
$10: 41: 49$ \\
\end{tabular} \\
\hline B198405131245A & 1984-05-13 & 12:45:53 \\
\hline E198405241956A & $1984-05-24$ & 19:56:04 \\
\hline E198406292318A & 1984-06-29 & 23:18:34 \\
\hline E198411050756A & 1984-11-05 & 07:56:01 \\
\hline T198501162335A & $1985-01-16$ & 23:35:58 \\
\hline E198504211813A & 1985-04-21 & 18:13:02 \\
\hline B198508150428A & $1985-08-15$ & 04:28:47 \\
\hline B198510271934A & $1985-10-27$ & 19:34:57 \\
\hline B198511212157A & $1985-11-21$ & 21:57:14 \\
\hline E198511212316A & $1985-11-21$ & 23:16:27 \\
\hline E198511211923A & $1985-11-21$ & 19:23:06 \\
\hline E198511222206A & $1985-11-22$ & 22:06:59 \\
\hline E198511222217A & $1985-11-22$ & 22:17:37 \\
\hline E198511231732A & $1985-11-23$ & $: 32: 30$ \\
\hline E198511230819A & $1985-11-23$ & 08:19:48 \\
\hline E198511241253A & $1985-11-24$ & 12:53:46 \\
\hline E198511241345A & $1985-11-24$ & 13:45:04 \\
\hline E198511241506A & 1985-11-24 & 15:06:42 \\
\hline E198511252349A A & $1985-11-25$ & 23:49:50 \\
\hline S198511251643A & $1985-11-25$ & 16:43:56 \\
\hline E198512211118A & $1985-12-21$ & 11:18:12 \\
\hline E198512210149A A & $1985-12-21$ & 01:49:39 \\
\hline E198512270948A & $1985-12-27$ & 09:48:42 \\
\hline S198601080027A & $1986-01-08$ & $: 27: 20$ \\
\hline E198601131348A & 1986-01-13 & \begin{tabular}{|l|}
$13: 48: 03$ \\
\end{tabular} \\
\hline S198601260733A A & 1986-01-26 & 07:33:56 \\
\hline E198603242213A & 1986-03-24 & 22:13:16 \\
\hline E198606072003A & $1986-06-07$ & 20:03:25 \\
\hline E198606072006A & $1986-06-07$ & 20:06:27 \\
\hline S198608291457A & $1986-08-29$ & 14:57:02 \\
\hline S198610011953A & $1986-10-01$ & 19:53:41 \\
\hline E198610181513A & $1986-10-18$ & 15:13:46 \\
\hline B198611251359A & $1986-11-25$ & 13:59:42 \\
\hline E198611271210A & $1986-11-27$ & 12:10:03 \\
\hline S198612160622A & $1986-12-16$ & 06:22:51 \\
\hline S198612241648A A & $1986-12-24$ & 16:48:05 \\
\hline E198701070039A & 1987-01-07 & 00:39:27 \\
\hline R198701280533A & $1987-01-28$ & 05:33:21 \\
\hline E198702102120A & $1987-02-10$ & 21:20:00 \\
\hline E198704240230A & 1987-04-24 & 02:30:00 \\
\hline S198705022043A & $1987-05-02$ & 20:43:53 \\
\hline & 87-05-08 & 10:00 \\
\hline E198705241023A & $1987-05-24$ & 10:23:00 \\
\hline S198707031021A & 1987-07-03 & 10:21:58 \\
\hline S198700051312A & 1987-07-05 & 13:12:36 \\
\hline R198708130722A & 1987-08-13 & 07:22:09 \\
\hline S198709041642A & 1987-09-04 & 16:42:00 \\
\hline E198801041851A & 1988-01-04 & 18:51:54 \\
\hline R198801081305A & 1988-01-08 & 13:05:46 \\
\hline & 1988-01-09 & :10:52 \\
\hline B1988001090102A & 1988-01-09 & 01:02:47 \\
\hline & 88-03-16 & 2:05 \\
\hline E198803191152A & 1988-03-19 & 11:52:35 \\
\hline S198804261917A & $1988-04-26$ & 19:17:56 \\
\hline B198804260053A & 1988-04-26 & 00:53:45 \\
\hline E198808040357A & 1988-08-04 & 03:57:00 \\
\hline & $1988-08-23$ & $: 38: 47$ \\
\hline E198812161135A & $1988-12-16$ & 11:35:05 \\
\hline B198901031652A & 1989-01-03 & $16: 52: 19$ \\
\hline T198901060526A & 1989-01-06 & 05:26:58 \\
\hline S1989091 & 1989-09-13 & 50.50 \\
\hline T198909242127A & 1989-09-24 & 21:27:54 \\
\hline & 1989-12-06 & 33:0 \\
\hline S198912220648A & $1989-12-22$ & : :48:13 \\
\hline E198912 & $1989-12-26$ & :59:59 \\
\hline 0445A & 0-01-24 & 45:05 \\
\hline & 1990-02-01 & 24:14 \\
\hline E199002110700A & 1990-02-11 & 07:00:37 \\
\hline E199002 & $1990-02-15$ & 5600 \\
\hline S199002182010A & $1990-02-18$ & 20:10:48 \\
\hline & & \\
\hline T199004032202A & 1990-04-03 & 22:02:37 \\
\hline E199004150750A & 1990-04-15 & 07:50:36 \\
\hline & & \\
\hline B199005050721A & 1990-05-05 & 07:21:19 \\
\hline & $-07-31$ & :50:52 \\
\hline 99009031048A & 1990-09-03 & 48:33 \\
\hline
\end{tabular}




\begin{tabular}{|c|c|c|}
\hline ID event & date & time \\
\hline R199010290816A & 1990-10-29 & 08:16:14 \\
\hline E199011270451A & 90-11-27 & :51:03 \\
\hline B199011270437A & 1990-11-27 & 04:37:57 \\
\hline B199012130024A & $1990-12-13$ & 00:24:24 \\
\hline B199012140321A & $1990-12-14$ & 03:21:26 \\
\hline S199102111543A & 1991-02-11 & 15:43:43 \\
\hline B199102260725A & 1991-02-26 & 07:25:47 \\
\hline R199105261226A & $1991-05-26$ & 12:26:00 \\
\hline S199109081945A & 1991-09-08 & 19:45:22 \\
\hline E199110310931A & 1991-10-31 & 09:31:00 \\
\hline E199111200154A & 1991-11-20 & 01:54:00 \\
\hline R199204061308A & 1992-04-06 & 13:08:34 \\
\hline B199204251246A & 1992-04-25 & 12:46:17 \\
\hline S1992005080644A & 1992-05-08 & 06:44:39 \\
\hline E199205111334A & 1992-05-11 & 13:34:00 \\
\hline S199206071145A & 1992-06-07 & 11:45:59 \\
\hline S199207160538A & 1992-07-16 & 05:38:53 \\
\hline E199210300538A & $1992-10-30$ & 05:38:27 \\
\hline S199306011951A & 1993-06-01 & 19:51:10 \\
\hline S199306051916A & $1993-06-05$ & 19:16:16 \\
\hline R199306261747A & 1993-06-26 & 17:47:53 \\
\hline E199307171035A & 1993-07-17 & 10:35:00 \\
\hline E199308310016A & 1993-08-31 & 00:16:00 \\
\hline B199309101602A & $1993-09-10$ & 16:02:32 \\
\hline E199310010413A & 1993-10-01 & 04:13:09 \\
\hline E199311271326A & $1993-11-27$ & 13:26:00 \\
\hline T199312242153A & 1993-12-24 & 21:53:19 \\
\hline B199401051324A & 1994-01-05 & 13:24:11 \\
\hline E199401200705A & 1994-01-20 & 07:05:42 \\
\hline E199401200659A & 1994-01-20 & 06:59:13 \\
\hline E199402011757A & 1994-02-01 & 17:57:00 \\
\hline E199402251603A & 94-02-25 & 16:03:05 \\
\hline R199403212140A & 1994-03-21 & 21:40:57 \\
\hline S199404202125A & 1994-04-20 & 21:25:25 \\
\hline T199409170456A & 1994-09-17 & 04:56:26 \\
\hline E199412140856A & 1994-12-14 & 08:56:00 \\
\hline A199501072030A & 1995-01-07 & 20:30:47 \\
\hline E199503040158A & 1995-03-04 & 01:58:13 \\
\hline E199504210802A & 1995-04-21 & 08:02:57 \\
\hline E199505131016A & 1995-05-13 & 10:16:01 \\
\hline S199505221250A & 1995-05-22 & 12:50:31 \\
\hline E199505221116A & $1995-05-22$ & 11:16:53 \\
\hline R199505290652A & $1995-05-29$ & 06:52:27 \\
\hline S199507150645A & 1995-07-15 & 06:45:22 \\
\hline E199507231844A & 1995-07-23 & 18:44:00 \\
\hline S199508241727A & $1995-08-24$ & 17:27:34 \\
\hline S199508250927A & $1995-08-25$ & $: 27: 21$ \\
\hline B199509221120A & 1995-09-22 & 11:20:10 \\
\hline B199509282344A & $1995-09-28$ & 23:44:41 \\
\hline B199509301014A & $1995-09-30$ & 10:14:30 \\
\hline S199510100654A & $1995-10-10$ & 06:54:22 \\
\hline S199512312129A & 1995-12-31 & 21:29:47 \\
\hline E199601211217A & 1996-01-21 & 12:17:00 \\
\hline B199602011757A A & 1996-02-01 & 17:57:55 \\
\hline T199602201412A & 1996-02-20 & 14:12:00 \\
\hline E199602201409A & 1996-02-20 & 14:09:00 \\
\hline R199602250120A & 1996-02-25 & 01:20:01 \\
\hline E199603310608A & 1996-03-31 & 06:08:00 \\
\hline R199604031304A & $1996-04-03$ & 13:04:34 \\
\hline & 36-07-15 & 13:30 \\
\hline C199609052044A & 1996-09-05 & 20:44:09 \\
\hline E199609050244A & 1996-09-05 & 02:44:00 \\
\hline S199609052143A & 1996-09-05 & :43:31 \\
\hline B199609091557A & 1996-09-09 & 15:57:05 \\
\hline B199609171345A & 1996-09-17 & 13:45:22 \\
\hline S199610151218A & $1996-10-15$ & 12:18:24 \\
\hline B199610150955A & 1996-10-15 & 09:55:59 \\
\hline s19961 & $996-10-20$ & :06:55 \\
\hline E199611102323A & $1996-11-10$ & 23:23:00 \\
\hline & 96-12-14 & 18:44 \\
\hline S199612210846A & $1996-12-21$ & 08:46:01 \\
\hline R19970112 & 1997-01-12 & 12:10:51 \\
\hline R199701191942A & 1997-01-19 & 19:42:38 \\
\hline E199701270046A & 1997-01-27 & 00:46:17 \\
\hline & 1997-03-19 & :10:49 \\
\hline R199703250046A & 1997-03-25 & 00:46:13 \\
\hline E199704260730A & $1997-04-26$ & 07:30:18 \\
\hline R199707200339A & 1997-07-20 & 03:39:11 \\
\hline E199707301606A & 1997-07-30 & 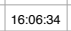 \\
\hline R199709032207A & 1997-09-03 & 22:07:29 \\
\hline & 77-09-16 & :03:59 \\
\hline R199709261330A & 1997-09-26 & 13:30:51 \\
\hline 60033A & 1997-09-26 & :33:12 \\
\hline R1997092 & 7-09-26 & $54 \cdot 27>$ \\
\hline C19970 & $1997-09-26$ & 40:26 \\
\hline E199709260947A & 1997-09-26 & 09:47:38 \\
\hline R199709 & 1997-09-27 & \\
\hline R199709271956A & $1997-09-27$ & 19:56:43 \\
\hline & & \\
\hline E199710021059A & 1997-10-02 & 10:59:56 \\
\hline R199710021938A & $1997-10-02$ & 19:38:02 \\
\hline & & \\
\hline R199710040649A & 1997-10-04 & : :49:59 \\
\hline & 10-04 & 07:26 \\
\hline R & $97-10-04$ & \\
\hline
\end{tabular}




\begin{tabular}{|c|c|c|}
\hline ID event & date & time \\
\hline R199710041613A & 1997-10-04 & 16:13:33 \\
\hline $99710062324 \mathrm{~A}$ & 7-10-06 & $: 24: 53$ \\
\hline R199710070124A & 1997-10-07 & 01:24:33 \\
\hline R199710070509A & 1997-10-07 & 05:09:56 \\
\hline R199710122131A & 1997-10-12 & 21:31:07 \\
\hline R199710121108A & $1997-10-12$ & 11:08:36 \\
\hline R199710131309A & $1997-10-13$ & 13:09:21 \\
\hline R199710131101A A & 1997-10-13 & 11:01:46 \\
\hline R199710141523A & $1997-10-14$ & 15:23:10 \\
\hline R199710152253A & $1997-10-15$ & 22:53:10 \\
\hline R199710161731A & 1997-10-16 & 17:31:43 \\
\hline R199710160223A & $1997-10-16$ & |02:23:37 \\
\hline R199710160452A & $1997-10-16$ & 04:52:55 \\
\hline R199710161200A & $1997-10-16$ & 12:00:31 \\
\hline R199710171422A & $1997-10-17$ & 14:22:45 \\
\hline R199710191600A & $1997-10-19$ & 16:00:16 \\
\hline R199710250308A & $1997-10-25$ & 03:08:06 \\
\hline R199710310423A & $1997-10-31$ & 04:23:42 \\
\hline R199711091907A & 1997-11-09 & 19:07:32 \\
\hline R199711301124A & $1997-11-30$ & 11:24:42 \\
\hline B199712021922A & 1997-12-02 & 19:22:44 \\
\hline R199712241753A & 1997-12-24 & 17:53:08 \\
\hline R199712311602A & 1997-12-31 & 16:02:15 \\
\hline R199801171232A & 1998-01-17 & 12:32:51 \\
\hline R199802070059A A & 1998-02-07 & 00:59:48 \\
\hline R199802261209A & 1998-02-26 & 12:09:19 \\
\hline R1998003010722A & 1998-03-01 & 07:22:41 \\
\hline R199803132307A & 1998-03-13 & 23:07:29 \\
\hline R199803131515A & 1998-03-13 & 15:15:56 \\
\hline R199803211645A & 1998-03-21 & 16:45:09 \\
\hline R199803261626A & 1998-03-26 & 16:26:13 \\
\hline R199804030726A & $1998-04-03$ & :26:36 \\
\hline R199804030759A A & 1998-04-03 & 07:59:53 \\
\hline R199800051552A & 1998-04-05 & 15:52:20 \\
\hline B199804121055A & 1998-04-12 & 10:55:32 \\
\hline R199805060251A & 1998-05-06 & 02:51:59 \\
\hline R199805161055A & $1998-05-16$ & 10:55:12 \\
\hline B199805181719A & 1998-05-18 & 17:19:04 \\
\hline R199806022311A & 1998-06-02 & 23:11:22 \\
\hline R199806200225A & 1998-06-20 & 02:25:47 \\
\hline R199806211259A & 1998-06-21 & 12:59:04 \\
\hline R199806210859A & $1998-06-21$ & :59:47 \\
\hline R199806250032A & $1998-06-25$ & 00:32:53 \\
\hline R199808150518A & 1998-08-15 & 05:18:08 \\
\hline R199808310231A & 1998-08-31 & 02:31:04 \\
\hline B199809091127A & 1998-09-09 & 11:27:59 \\
\hline R199809140524A & $1998-09-14$ & :24:47 \\
\hline E199809222323A & 1998-09-22 & 23:23:00 \\
\hline R199901252245A & 1999-01-25 & 22:45:57 \\
\hline R199902141145A & 1999-02-14 & 11:45:54 \\
\hline E199902140558A & 1999-02-14 & 05:58:00 \\
\hline R1999007071716A & 1999-07-07 & 17:16:10 \\
\hline R199909141547A & 1999-09-14 & 15:47:24 \\
\hline R199910101535A A & 1999-10-10 & 15:35:51 \\
\hline R199910230206A & 1999-10-23 & 02:06:00 \\
\hline R199910300708A & 1999-10-30 & 07:08:09 \\
\hline R199911280059A & $1999-11-28$ & \begin{tabular}{|l|l|l}
$00: 59: 47$ \\
\end{tabular} \\
\hline R199912292042A & 1999-12-29 & \begin{tabular}{|l|l|}
$20: 42: 36$ \\
\end{tabular} \\
\hline R199912301834A & $1999-12-30$ & 18:34:37 \\
\hline & 19-12-31 & 55:55 \\
\hline R200003111035A & 2000--03-11 & 10:35:26 \\
\hline R200003300931A & 2000-03-30 & 09:31:31 \\
\hline R200004011808A & 2000-04-01 & 18:08:04 \\
\hline R200004061740A & 2000-04-06 & 17:40:38 \\
\hline R200004261337A & $2000-04-26$ & 13:37:48 \\
\hline R200004262225A & $2000-04-26$ & 22:25:07 \\
\hline R200004281741A & $2000-04-28$ & 17:41:02 \\
\hline & 2000-05-02 & 08:48:47 \\
\hline R200005052042A & 2000-05-05 & 20:42:01 \\
\hline & 000-05-06 & 05:00 \\
\hline H200005070044A & $2000-05-07$ & 00:44:06 \\
\hline R2000050 & 2000-05-08 & 12:30:00 \\
\hline R200005091751A & 2000-05-09 & 17:51:19 \\
\hline R200005101652A & $2000-05-10$ & 16:52:09 \\
\hline & 00-05-11 & $: 57: 15$ \\
\hline R200005121126A & $2000-05-12$ & 11:26:38 \\
\hline R200006180742A & 2000-06-18 & 07:42:02 \\
\hline R200006221216A & $2000-06-22$ & 12:16:31 \\
\hline & 000-06-27 & 0 \\
\hline R200006270732A & $2000-06-27$ & 07:32:31 \\
\hline & 00-08-01 & $34: 2$ \\
\hline R200008211714A & $2000-08-21$ & 17:14:27 \\
\hline R200009020516A & 2000-09-02 & :16:59 \\
\hline & $-10-03$ & 0 \\
\hline & 2000-10-08 & 43:48 \\
\hline R200011090300A & 2000-11-09 & 03:00:03 \\
\hline & 000-12-16 & 0 \\
\hline H200101042220A & 2001-01-04 & 22:20:59 \\
\hline & & \\
\hline R200104170042A & 2001-04-17 & 00:42:36 \\
\hline R200104221356A & $2001-04-22$ & 13:56:35 \\
\hline & & \\
\hline R200105260602A & 2001-05-26 & 06:02:20 \\
\hline & 01-07-.02 & 04:43 \\
\hline R200107171506A & 001-07-17 & 66:15 \\
\hline
\end{tabular}




\begin{tabular}{|c|c|c|}
\hline ID event & date & time \\
\hline R200107182247A & 2001-07-18 & 22:47:10 \\
\hline $200108121422 A$ & 01-08-12 & :22:25 \\
\hline R200110181808A & 2001-10-18 & 18:08:40 \\
\hline R200110181102A & 2001-10-18 & 11:02:44 \\
\hline R200111070940A & 2001-11-07 & 09:40:45 \\
\hline R200111251934A A & $2001-11-25$ & 19:34:19 \\
\hline R200111260056A & $2001-11-26$ & 0:56:55 \\
\hline R200201310848A & 2002-01-31 & 08:48:38 \\
\hline R200202140318A & 2002-02-14 & 03:18:01 \\
\hline R200202211436A & 2002-02-21 & 14:36:11 \\
\hline R200204050452A & 2002-04-05 & 04:52:23 \\
\hline R200204080120A & 2002-04-08 & :20:47 \\
\hline R200204102115A & 2002-04-10 & 21:15:11 \\
\hline R200204170642A & 2002-04-17 & 06:42:54 \\
\hline R200204182056A & 2002-04-18 & 20:56:47 \\
\hline R200205281945A & $2002-05-28$ & 19:45:11 \\
\hline R200206082013A & 2002-06-08 & 20:13:11 \\
\hline R200206182223A & 2002-06-18 & 22:23:37 \\
\hline R200206240120A & 2002-06-24 & 01:20:36 \\
\hline B2002090600121A & 2002--09-06 & 01:21:28 \\
\hline R200209060145A & 2002-09-06 & 01:45:30 \\
\hline R200209090606A & 2002-09-09 & 06:06:37 \\
\hline R200209100232A & $2002-09-10$ & 02:32:51 \\
\hline R200209202306A & $2002-09-20$ & 23:06:03 \\
\hline R200209270610A & $2002-09-27$ & 06:10:44 \\
\hline R200209280246A & 2002-09-28 & 02:46:46 \\
\hline G200210022257A & 2002-10-02 & :57:25 \\
\hline R200210231101A & 2002-10-23 & 11:01:28 \\
\hline R200210270250A & $2002-10-27$ & 02:50:26 \\
\hline R200210270732A & 2002-10-27 & 07:32:08 \\
\hline R200210291002A & 2002-10-29 & 10:02:21 \\
\hline R200210291639A & 002-10-29 & : :39:47 \\
\hline B200210311032A & 2002-10-31 & 10:32:58 \\
\hline B200211011509A & 2002-11-01 & 15:09:00 \\
\hline R200211011721A & 2002-11-01 & 17:21:31 \\
\hline R200211120927A & 2002-11-12 & 09:27:47 \\
\hline R200212090935A & 2002-12-09 & 09:35:04 \\
\hline R200301262015A & 2003-01-26 & 20:15:03 \\
\hline R200301261957A & 2003-01-26 & 19:57:03 \\
\hline R200303271610A & 2003-03-27 & 16:10:36 \\
\hline R200303291742A & 2003-03-29 & 17:42:15 \\
\hline R200303300056A & 2003-03-30 & :56:24 \\
\hline R200303301109A & 2003-03-30 & 11:09:59 \\
\hline R200303311936A & 2003-03-31 & 19:36:29 \\
\hline R200304032230A & 2003-04-03 & 22:30:11 \\
\hline R200304110926A & 2003-04-11 & 09:26:58 \\
\hline R200304260820A & 2003-04-26 & $: 20: 43$ \\
\hline R200304291513A & 2003-04-29 & 15:13:06 \\
\hline R200305050616A & 2003-05-05 & 06:16:38 \\
\hline R200305100642A & 2003-05-10 & 06:42:49 \\
\hline R200306011545A & 2003-06-01 & 15:45:18 \\
\hline R200306160827A & 2003-06-16 & 08:27:51 \\
\hline R200306210900A & 2003-06-21 & 09:00:20 \\
\hline R200307071508A & 2003-07-07 & 15:08:12 \\
\hline R200307211315A & 2003-07-21 & 13:15:57 \\
\hline R200308021018A & $2003-08-02$ & 10:18:38 \\
\hline R200309142142A & 2003-09-14 & 21:42:53 \\
\hline R200309201150A & 2003-09-20 & \begin{tabular}{|l|l|}
$11: 50: 49$ \\
\end{tabular} \\
\hline H200312060018A & 2003-12-06 & $00: 18: 55$ \\
\hline 0031 & 2003-12-07 & 20:33 \\
\hline R200312300531A & 2003-12-30 & 05:31:38 \\
\hline H200403030213A & 2004-03-03 & 02:13:26 \\
\hline R200403231238A & 2004-03-23 & 12:38:30 \\
\hline R200405051339A A & 2004-05-05 & 13:39:43 \\
\hline R200405231519A & $2004-05-23$ & 15:19:08 \\
\hline R200407121304A & 2004-07-12 & 13:04:05 \\
\hline R200409141809A & 2004-09-14 & 18:09:26 \\
\hline & 20 & 59:40 \\
\hline R200411250621A & 2004-11-25 & 06:21:19 \\
\hline R & 04-12-03 & 1::14 \\
\hline R200412180912A & $2004-12-18$ & 09:12:48 \\
\hline S200502072005A & 2005-02-07 & 20:05:37 \\
\hline S200502072046A A & 2005--02-07 & 20:46:26 \\
\hline S200502121213A & 2005-02-12 & 12:13:45 \\
\hline & 2005-03-11 & :05:23 \\
\hline S200504181059A & 2005-04-18 & 10:59:18 \\
\hline S200505192319A & 2005-05-19 & 23:19:56 \\
\hline S200507032344A & 2005-07-03 & 23:44:42 \\
\hline B200507101310A & 2005-07-10 & 0.1 \\
\hline C200507260332A & 2005-07-26 & 03:32:15 \\
\hline & 305-08-22 & :02:08 \\
\hline S200509081127A & 2005-09-08 & 11:27:17 \\
\hline S20050 & $2005-09-27$ & :25:35 \\
\hline & s-11-21 & :57:41 \\
\hline & 006-01-28 & $: 26: 05$ \\
\hline S200602270434A & $2006-02-27$ & 04:34:01 \\
\hline S200604 & 2006-04-10 & อ2? \\
\hline S200604162115A & 2006-04-16 & 21:15:01 \\
\hline & & \\
\hline S200604172142A & 2006-04-17 & 21:42:57 \\
\hline S200605102133A & 2006-05-10 & 21:33:06 \\
\hline & & \\
\hline S2006005290220A & 2006-05-29 & 02:20:06 \\
\hline & 05-30 & 30:40 \\
\hline 0006061 & & \\
\hline
\end{tabular}




\begin{tabular}{|c|c|c|}
\hline ID event & date & time \\
\hline S200606221934A & $2006-06-22$ & \begin{tabular}{|l|l|}
$19: 34: 58$ \\
\end{tabular} \\
\hline S200608060749A & 06-08-06 & 07:49:46 \\
\hline S200608082120A & 2006-08-08 & 21:20:09 \\
\hline S200608090210A & 2006-08-09 & 02:10:01 \\
\hline S200609071531A & 2006-09-07 & 15:31:43 \\
\hline S200610041734A & 2006-10-04 & 17:34:20 \\
\hline S200610190934A & 2006-10-19 & 09:34:51 \\
\hline S200610210704A & $2006-10-21$ & 07:04:10 \\
\hline C200610261428A & $2006-10-26$ & 14:28:37 \\
\hline S200611031050A & 2006-11-03 & 10:50:52 \\
\hline S200611051715A & 2006-11-05 & 17:15:00 \\
\hline S200611071113A & 2006-11-07 & 11:13:36 \\
\hline S200611240437A & $2006-11-24$ & 04:37:40 \\
\hline S200612101103A & 2006-12-10 & 11:03:42 \\
\hline S200612191458A & 2006-12-19 & 14:58:06 \\
\hline S200702031049A & 2007-02-03 & 10:49:14 \\
\hline S200702050830A & 2007-02-05 & 08:30:05 \\
\hline S200702220603A & 2007-02-22 & 06:03:01 \\
\hline S200703261355A & 2007-03-26 & 13:55:25 \\
\hline C200704160738A & 2007-04-16 & 07:38:53 \\
\hline S200705090603A & 2007-05-09 & 06:03:49 \\
\hline C200707042355A & 2007-07-04 & 23:55:32 \\
\hline S200707301905A & $2007-07-30$ & 19:05:43 \\
\hline S200708181404A & $2007-08-18$ & 14:04:08 \\
\hline C200709050508A & 2007-09-05 & 05:08:15 \\
\hline S200710181541A A & 2007-10-18 & 15:41:20 \\
\hline S200710210355A & 2007-10-21 & 03:55:35 \\
\hline S200711240336A & 2007-11-24 & 03:36:04 \\
\hline S200712010714A & 2007-12-01 & 07:14:25 \\
\hline S200802200806A & 2008-02-20 & 08:06:19 \\
\hline S200802210500A & 2008-02-21 & 05:00:08 \\
\hline S200803010843A & 2008-03-01 & 08:43:46 \\
\hline S200803010743A & 2008-03-01 & 07:43:13 \\
\hline S200803010808A & 2008-03-01 & 08:08:09 \\
\hline S200803050408A & 2008-03-05 & 04:08:21 \\
\hline C200803191438A & 2008-03-19 & 14:38:57 \\
\hline S200803260919A & $2008-03-26$ & 09:19:31 \\
\hline S200804081720A & 2008-04-08 & 17:20:03 \\
\hline C200806021736A & $2008-06-02$ & 17:36:12 \\
\hline S200810240306A & $2008-10-24$ & 03:06:40 \\
\hline S200811201409A A & $2008-11-20$ & 14:09:19 \\
\hline S200811282339A & $2008-11-28$ & $: 39: 21$ \\
\hline C200812172157A & 2008-12-17 & 21:57:44 \\
\hline S200812232158A & 2008-12-23 & 21:58:25 \\
\hline C200812231524A & $2008-12-23$ & 15:24:22 \\
\hline S200901041530A & 2009-01-04 & 15:30:30 \\
\hline S200903190827A & 2009-03-19 & 08:27:54 \\
\hline S200903251223A & 2009-03-25 & 12:23:25 \\
\hline S200903301338A & 2009-03-30 & 13:38:38 \\
\hline S2009004052048A & 2009-04-05 & 20:48:56 \\
\hline S2009004052020A & 2009-04-05 & 20:20:52 \\
\hline C200904060132A & 2009-04-06 & 01:32:39 \\
\hline C200904060717A & 2009-04-06 & 07:17:10 \\
\hline S200900061638A & 2009-04-06 & 16:38:09 \\
\hline S200904060356A & 2009-04-06 & 03:56:48 \\
\hline C200904060237A & 2009-04-06 & 02:37:05 \\
\hline C200904062315A & 2009-04-06 & 23:15:37 \\
\hline C200904071747A & 2009-04-07 & 17:47:37 \\
\hline C200904070926A & 2009-04-07 & 09:26:28 \\
\hline & 09--04-07 & $34: 29$ \\
\hline S2009004080427A & 2009-04-08 & 04:27:42 \\
\hline S200904082256A & 2009-04-08 & 22:56:50 \\
\hline S200900090314A & 2009-04-09 & :14:52 \\
\hline S200904090432A & 2009-04-09 & 04:32:44 \\
\hline C200904090052A & 2009-04-09 & 00:52:59 \\
\hline C200904091938A & $2009-04-09$ & 19:38:16 \\
\hline S200904100322A & 2009-04-10 & 03:22:23 \\
\hline & 2009-04-13 & 14:24 \\
\hline S200904142017A & 2009--04-14 & 20:17:27 \\
\hline S20090 & 009-04-15 & 33:08 \\
\hline S200904232149A & $2009-04-23$ & 21:49:00 \\
\hline S200904231514A & 2009-04-23 & 15:14:08 \\
\hline S200905072127A A & 2009-05-07 & 21:27:12 \\
\hline S200906211120A & 2009-06-21 & 11:20:04 \\
\hline 009 & 2009-06-22 & :58:40 \\
\hline S200907031103A & 2009-07-03 & 11:03:07 \\
\hline S200907120838A & 2009-07-12 & 08:38:51 \\
\hline C200908211339A & 2009--08-21 & 13:39:57 \\
\hline c20 & 2009-09-07 & 2 \\
\hline S200909200350A & $2009-99-20$ & 03:50:15 \\
\hline & $2009-09-24$ & 14:5 \\
\hline S200910191008A & 2009-10-19 & 10:08:50 \\
\hline S200912 & $2009-12-15$ & $: 11: 58$ \\
\hline & $-12-19$ & $01: 18$ \\
\hline & $2010-01-12$ & $25: 10$ \\
\hline S201001121335A & 2010-01-12 & 13:35:47 \\
\hline & 2010-02-11 & 25.59 \\
\hline S201004022004A & 2010-04-02 & 20:04:47 \\
\hline & & \\
\hline S201004150147A & 2010-04-15 & 01:47:37 \\
\hline S201006041844A & 2010-06-04 & 18:44:53 \\
\hline & & \\
\hline C201006202313A & 2010-06-20 & 23:13:52 \\
\hline & 10-07-09 & 15:10 \\
\hline S201008161254A & 010-08-16 & 54:47 \\
\hline
\end{tabular}




\begin{tabular}{|c|c|c|}
\hline ID event & date & time \\
\hline C201008221022A & $2010-08-22$ & 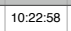 \\
\hline S201008280708A & 10-08-28 & 07:08:03 \\
\hline S201009050707A & 2010-09-05 & 07:07:23 \\
\hline S201009171220A & $2010-09-17$ & 12:20:18 \\
\hline S201010132243A & $2010-10-13$ & 22:43:14 \\
\hline S201010150521A & 2010-10-15 & 05:21:19 \\
\hline S201011131824A & 2010-11-13 & 18:24:59 \\
\hline S201101220048A & 2011-01-22 & 00:48:49 \\
\hline S201101240126A & 2011-01-24 & 01:26:39 \\
\hline S201101291741A & 2011-01-29 & 17:41:37 \\
\hline S201104241302A & 2011-04-24 & 13:02:12 \\
\hline S201104282330A & 2011-04-28 & 23:30:44 \\
\hline S201105061512A & 2011-05-06 & 15:12:35 \\
\hline S201105191450A & 2011-05-19 & 14:50:52 \\
\hline S201105242203A & $2011-05-24$ & 22:03:55 \\
\hline S201106091616A & 2011-06-09 & 16:16:36 \\
\hline S201106170531A & 2011-06-17 & 05:31:08 \\
\hline S201106232202A & $2011-06-23$ & 22:02:46 \\
\hline S201107021443A & 2011-07-02 & 14:43:08 \\
\hline S201107060908A & 2011-07-06 & 09:08:38 \\
\hline C201107071921A & 2011-07-07 & 19:21:48 \\
\hline S201107120715A & $2011-07-12$ & 07:15:08 \\
\hline S201107120653A & 2011-07-12 & 06:53:22 \\
\hline S201107121920A & $2011-07-12$ & 19:20:57 \\
\hline S201107130955A & 2011-07-13 & 09:55:12 \\
\hline S201107171830A & 2011-07-17 & 18:30:23 \\
\hline S201107251231A & $2011-07-25$ & 12:31:20 \\
\hline S201108151308A & 2011-08-15 & 13:08:35 \\
\hline S201109251317A & 2011-09-25 & 13:17:10 \\
\hline S201110200611A & 2011-10-20 & 06:11:00 \\
\hline S201110290413A & 2011-10-29 & 04:13:34 \\
\hline S201111150459A & 2011-11-15 & :59:00 \\
\hline S201112121704A & 2011-12-12 & 17:04:43 \\
\hline S201112181501A & 2011-12-18 & 15:01:03 \\
\hline S201201242354A & 2012-01-24 & 23:54:46 \\
\hline S201201250806A & $2012-01-25$ & 08:06:37 \\
\hline C201201271453A & $2012-01-27$ & 14:53:14 \\
\hline S201202252034A & 2012-02-25 & 20:34:34 \\
\hline S201202262237A & 2012-02-26 & 22:37:55 \\
\hline S201203040347A & 2012-03-04 & 03:47:39 \\
\hline S201203051515A & 2012-03-05 & 15:15:06 \\
\hline S201203150329A & 2012-03-15 & $: 29: 05$ \\
\hline S201204130621A & 2012-04-13 & 06:21:35 \\
\hline S201205192313A & 2012-05-19 & 23:13:27 \\
\hline C201205201318A & $2012-05-20$ & 13:18:02 \\
\hline C201205200302A & $2012-05-20$ & 03:02:50 \\
\hline S201205200913A & 2012-05-20 & 09:13:21 \\
\hline S201205201737A & 2012-05-20 & 17:37:14 \\
\hline C201205200203A & 2012-05-20 & 02:03:51 \\
\hline S201205211637A & 2012-05-21 & 16:37:31 \\
\hline S201205232141A & $2012-05-23$ & 21:41:18 \\
\hline S201205251314A & 2012-05-25 & 13:14:05 \\
\hline S201205271818A & $2012-05-27$ & 18:18:45 \\
\hline S201205280106A & $2012-05-28$ & 01:06:27 \\
\hline C201205290700A & 2012-05-29 & 07:00:03 \\
\hline S201205290827A & 2012-05-29 & 08:27:23 \\
\hline S201205291100A & 2012-05-29 & 11:00:25 \\
\hline C201205291055A & 2012-05-29 & 10:55:57 \\
\hline S201205290825A & 2012-05-29 & 08:25:51 \\
\hline & 12-05-31 & 58:21 \\
\hline S201206031920A & $2012-06-03$ & 19:20:43 \\
\hline S201206060408A & 2012-06-06 & 04:08:31 \\
\hline S201206090204A & 2012-06-09 & :04:56 \\
\hline S201206120148A & 2012-06-12 & 01:48:36 \\
\hline C201206182209A & $2012-06-18$ & 22:09:49 \\
\hline S201207041112A & 2012-07-04 & 11:12:12 \\
\hline S201207041327A & 2012-07-04 & 13:27:12 \\
\hline & 12-07-27 & :12:22 \\
\hline S201207300901A & 2012-07-30 & 09:01:17 \\
\hline & 12-08-03 & 77:27 \\
\hline S201208120121A & $2012-08-12$ & 01:21:36 \\
\hline S201208130730A & 2012-08-13 & 07:30:51 \\
\hline S201208282312A & $2012-08-28$ & 23:12:15 \\
\hline S201209042243A & 2012-09-04 & 22:43:43 \\
\hline 0120 & 12-09-29 & 39:44 \\
\hline S201210030920A & $2012-10-03$ & 09:20:43 \\
\hline S201210031441A & 2012-10-03 & 14:41:30 \\
\hline S201210161510A & 2012-10-16 & 15:10:59 \\
\hline & $12-10-25$ & 2 \\
\hline S201211122318A & $2012-11-12$ & 23:18:42 \\
\hline & 2-11-13 & $06: 3$ \\
\hline C201211132324A & 2012-11-13 & 23:24:10 \\
\hline & 12-11-22 & :25:52 \\
\hline $9910 \mathrm{~A}$ & 2-11-22 & 10.4 \\
\hline & 12-12-13 & $39: 27$ \\
\hline S201301040750A & 2013-01-04 & 07:50:06 \\
\hline & 2013-01-25 & 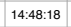 \\
\hline S201302021335A & 2013-02-02 & 13:35:34 \\
\hline & & \\
\hline C201302162116A & 2013-02-16 & 21:16:09 \\
\hline S201303241547A & 2013-03-24 & 15:47:22 \\
\hline & & \\
\hline S201306162005A & 2013-06-16 & 20:05:00 \\
\hline & $-06-21$ & 10:33:57 \\
\hline S201306211212A & 13-06-21 & 2:39 \\
\hline
\end{tabular}




\begin{tabular}{|c|c|c|c|c|c|c|c|c|c|c|c|c|c|c|c|c|c|}
\hline ID event & date & time & latitude & longitude & $\begin{array}{l}\text { depth } \\
(\mathrm{kmm})\end{array}$ & $\mathrm{rr}$ & $\mathrm{tt}$ & $\mathrm{ff}$ & $\mathrm{rt}$ & if & tf & eigen & $\begin{array}{l}\text { plunge } \\
T\end{array}$ & $\begin{array}{l}\text { azimuth } \\
T\end{array}$ & $\begin{array}{c}\text { eigen } \\
B\end{array}$ & $\begin{array}{l}\text { plunge } \\
B\end{array}$ & $\underset{B}{\text { azimuth }}$ \\
\hline S201306220841A & 2013-06-22 & 08:41:08 & 40.21 & 19.58 & 10.0 & 2.880 & 0.600 & -3.480 & -0.230 & -4.580 & 1.190 & 5.400 & 60 & 109 & 0.620 & 12 & 357 \\
\hline S201306231501A & 2013-06-23 & 15:01:33 & 44.18 & 10.21 & 9.5 & -0.870 & 0.940 & -0.070 & -0.480 & 0.580 & 0.000 & 1.080 & 16 & 189 & 0.180 & 22 & 285 \\
\hline S201306301440A & 2013-06-30 & 14:40:08 & 44.17 & 10.20 & 9.8 & -1.220 & 1.260 & -0.030 & -1.360 & 0.300 & -0.280 & 1.930 & 24 & 192 & -0.090 & 0 & 282 \\
\hline S201307210307A & 2013-07-21 & 03:07:25 & 43.51 & 13.73 & 10.0 & 1.770 & -1.620 & -0.150 & -1.030 & -0.090 & 1.430 & 2.180 & 65 & 144 & 0.470 & 22 & 295 \\
\hline S201307210132A & 2013-07-21 & 01:32:24 & 43.53 & 13.74 & 10.0 & 5.120 & -3.200 & -1.930 & -0.400 & 2.350 & 4.850 & 5.990 & 67 & 293 & 1.730 & 22 & 136 \\
\hline S201307301258A & 2013-07-30 & 12:58:33 & 45.14 & 15.09 & 20.0 & 0.310 & -0.930 & 0.620 & -0.140 & -0.220 & -0.480 & 0.820 & 20 & 76 & 0.280 & 68 & 232 \\
\hline S201308152304A & 2013-08-15 & 23:04:59 & 38.15 & 14.91 & 10.0 & -0.140 & -0.200 & 0.340 & 1.080 & 0.520 & 0.140 & 1.180 & 41 & 318 & 0.110 & 14 & 61 \\
\hline S201308152306A & 2013-08-15 & 23:06:51 & 38.17 & 14.92 & 10.0 & -0.430 & -0.090 & 0.520 & 1.570 & 0.960 & 0.250 & 1.870 & 38 & 316 & 0.100 & 12 & 55 \\
\hline S201308220644A & 2013-08-22 & 06:44:52 & 43.58 & 13.69 & 10.0 & 5.260 & -1.880 & -3.380 & -2.030 & 2.430 & 2.650 & 6.130 & 75 & 228 & 0.120 & 1 & 322 \\
\hline S201308241718A & 2013-08-24 & 17:18:18 & 36.71 & 15.00 & 10.0 & 5.080 & -5.210 & 0.130 & -1.750 & -0.560 & 0.950 & 5.460 & 77 & 142 & 0.160 & 9 & 277 \\
\hline S201309020114A & 2013-09-02 & 01:14:14 & 39.51 & 15.40 & 326.0 & -1.280 & 0.640 & 0.640 & -0.590 & -5.890 & -4.390 & 7.180 & 29 & 59 & 0.520 & 36 & 173 \\
\hline S201309200206A & 2013-09-20 & 02:06:33 & 47.94 & 16.51 & 10.0 & -2.340 & 1.540 & 0.810 & 4.740 & -3.740 & 0.860 & 5.290 & 38 & 29 & 1.880 & 6 & 124 \\
\hline S201310181508A & 2013-10-18 & 15:08:32 & 35.61 & 10.98 & 10.0 & -0.090 & -0.570 & 0.660 & 0.140 & -0.900 & -1.150 & 1.740 & 25 & 62 & -0.370 & 58 & 201 \\
\hline S201310211937A A & 2013-10-21 & 19:37:03 & 35.60 & 10.93 & 10.0 & 0.140 & -0.460 & 0.320 & 1.840 & -2.410 & -5.960 & 7.190 & 23 & 47 & -1.130 & 67 & 219 \\
\hline S201311180758A & 2013-11-18 & 07:58:41 & 43.66 & 16.86 & 10.0 & 4.310 & -4.210 & -0.100 & -1.610 & 0.970 & 1.020 & 4.730 & 77 & 226 & 0.070 & 7 & 106 \\
\hline S201311211036A & 2013-11-21 & 10:36:49 & 44.91 & 9.00 & 10.0 & 0.840 & -0.750 & -0.090 & -0.420 & 0.900 & 0.050 & 1.440 & 59 & 253 & -0.450 & 19 & 128 \\
\hline S201312101833A & 2013-12-10 & 18:33:07 & 42.43 & 19.27 & 13.0 & 0.810 & -1.380 & 0.570 & -0.820 & -0.320 & 3.080 & 3.070 & 18 & 128 & 0.610 & 71 & 288 \\
\hline S201312150357A & 2013-12-15 & 03:57:33 & 36.67 & 14.94 & 15.0 & 1.200 & -0.900 & -0.300 & 0.270 & -1.080 & -1.240 & 2.020 & 53 & 61 & -0.070 & 36 & 221 \\
\hline S201312221006A & 2013-12-22 & 10:06:35 & 43.38 & 12.52 & 10.0 & -1.870 & 0.680 & 1.180 & 2.300 & -1.000 & -0.880 & 2.950 & 26 & 41 & 0.310 & 12 & 305 \\
\hline C201312291708A & 2013-12-29 & 17:08:43 & 41.37 & 14.45 & 11.0 & -6.410 & 3.790 & 2.620 & -0.790 & -2.210 & -3.420 & 6.730 & 4 & 41 & 0.430 & 18 & 133 \\
\hline S201312290654A & 2013-12-29 & $06: 54: 58$ & 43.12 & 17.30 & 10.0 & 3.170 & -2.150 & -1.010 & -0.250 & -1.290 & 1.670 & 3.630 & 70 & 113 & -0.220 & 19 & 309 \\
\hline S201401140435A & 2014-01-14 & 04:35:00 & 38.36 & 14.94 & 11.0 & 1.010 & -2.450 & 1.430 & 1.100 & 0.900 & -0.180 & 2.210 & 42 & 280 & 0.590 & 43 & 67 \\
\hline S201401140343A & 2014-01-14 & 03:43:42 & 38.37 & 14.92 & 12.0 & -0.160 & -1.610 & 1.770 & 0.060 & 0.540 & 0.030 & 1.910 & 15 & 271 & -0.300 & 75 & 82 \\
\hline S201401200712A & 2014-01-20 & 07:12:40 & 41.36 & 14.45 & 11.0 & -3.680 & 2.330 & 1.350 & 2.720 & -0.280 & -1.400 & 4.100 & 18 & 29 & 0.640 & 12 & 295 \\
\hline S201401200600A & 2014-01-20 & 06:00:15 & 41.39 & 19.48 & 10.0 & 0.850 & -0.190 & -0.660 & 0.590 & -0.570 & 0.180 & 1.220 & 65 & 35 & -0.200 & 10 & 147 \\
\hline S201401261906A & 2014-01-26 & 19:06:40 & 43.28 & 19.96 & 10.0 & 2.130 & -2.520 & 0.390 & -1.480 & 1.590 & 7.920 & 7.000 & 3 & 309 & 2.510 & 79 & 201 \\
\hline S201403081512A & 2014-03-08 & 15:12:32 & 41.48 & 19.43 & 10.0 & 1.840 & 1.020 & -2.860 & 1.270 & -1.870 & 1.070 & 2.940 & 60 & 23 & 1.000 & 21 & 154 \\
\hline S201403081506A & 2014-03-08 & 15:06:32 & 41.51 & 19.44 & 10.0 & 2.800 & -1.140 & -1.660 & -0.040 & -1.110 & -0.690 & 3.060 & 76 & 83 & -0.750 & 8 & 209 \\
\hline S201404051024A & 2014-04-05 & 10:24:46 & 38.82 & 17.18 & 64.0 & -0.580 & 0.250 & 0.330 & 0.100 & -0.780 & -1.560 & 2.010 & 14 & 48 & -0.480 & 57 & 160 \\
\hline C201404071926A & 2014-04-07 & 19:26:59 & 44.47 & 6.69 & 5.0 & -2.680 & -0.340 & 3.020 & -0.380 & 0.890 & 0.940 & 3.360 & 7 & 284 & -0.440 & 14 & 192 \\
\hline C201404220858A & 2014-04-22 & 08:58:26 & 45.61 & 14.18 & 25.0 & 0.080 & -0.800 & 0.720 & 0.030 & -0.220 & 0.740 & 1.060 & 11 & 112 & 0.050 & 78 & 318 \\
\hline S201405070620A & 2014-05--07 & 06:20:06 & 37.75 & 19.78 & 10.0 & 0.660 & -0.110 & -0.550 & 1.480 & -0.910 & -3.520 & 4.050 & 27 & 42 & -0.170 & 63 & 234 \\
\hline S201405190059A & 2014-05-19 & 00:59:20 & 40.94 & 19.81 & 10.0 & 0.610 & -3.780 & 3.170 & -1.570 & 2.440 & 1.090 & 4.650 & 31 & 271 & 0.080 & 51 & 134 \\
\hline S201405200443A & 2014-05-20 & 04:43:26 & 40.92 & 19.86 & 2.0 & 0.870 & -3.740 & 2.870 & -0.270 & 1.040 & 2.310 & 3.880 & 17 & 286 & 0.650 & 72 & 127 \\
\hline S201406061341A & 2014-06-06 & 13:41:38 & 39.90 & 16.09 & 10.0 & -2.390 & 0.260 & 2.120 & 2.470 & -1.430 & -0.660 & 3.300 & 24 & 57 & 0.690 & 17 & 319 \\
\hline S201408040554A & 2014-08-04 & 05:54:01 & 38.71 & 15.69 & 171.0 & 0.660 & -0.360 & -0.300 & -0.100 & -1.560 & -0.070 & 1.810 & 54 & 92 & -0.350 & 3 & 185 \\
\hline S201408281749A & 2014-08-28 & 17:49:20 & 45.67 & 10.70 & 10.0 & 0.960 & -0.590 & -0.360 & 0.180 & -0.440 & -0.580 & 1.160 & 66 & 59 & -0.090 & 23 & 227 \\
\hline S201408301816A & 2014-08-30 & 18:16:47 & 44.43 & 16.25 & 20.0 & -0.160 & -0.110 & 0.260 & 0.020 & -0.340 & 2.110 & 2.220 & 6 & 132 & -0.150 & 81 & 5 \\
\hline S201409051010A & 2014-09-05 & 10:10:19 & 39.19 & 18.76 & 10.0 & -1.310 & 0.640 & 0.670 & -0.790 & -0.160 & -0.650 & 1.390 & 10 & 220 & 0.270 & 21 & 126 \\
\hline S201409070956A & 2014-09--07 & 09:56:25 & 37.61 & 19.88 & 10.0 & 0.780 & -0.290 & -0.490 & 0.330 & -0.230 & 0.490 & 0.880 & 75 & 17 & 0.100 & 8 & 138 \\
\hline S201409071045A & 2014-09--07 & 10:45:01 & 44.11 & 10.66 & 12.0 & -3.800 & 1.170 & 2.630 & -2.000 & -4.070 & -1.800 & 4.790 & 22 & 75 & 1.840 & 23 & 175 \\
\hline S201409262338A & 2014-09-26 & 23:38:11 & 36.78 & 16.50 & 40.0 & -0.270 & 0.270 & 0.000 & -0.580 & 0.690 & -2.070 & 2.500 & 18 & 224 & -0.550 & 72 & 30 \\
\hline S201409301512A & 2014-09-30 & 15:12:41 & 43.06 & 18.35 & 10.0 & -2.790 & 0.840 & 1.940 & 1.150 & 0.790 & 1.450 & 3.230 & 12 & 306 & -0.080 & 9 & 38 \\
\hline S201410092258A & 2014-10-09 & 22:58:28 & 38.51 & 14.85 & 10.0 & 1.600 & -1.760 & 0.160 & -0.340 & 0.130 & -0.240 & 1.650 & 81 & 225 & 0.170 & 7 & 84 \\
\hline S201412141433A & 2014-12-14 & 14:33:31 & 40.46 & 15.17 & 307.0 & -3.470 & 3.580 & -0.110 & -1.000 & 0.440 & -1.820 & 4.480 & 8 & 202 & -0.850 & 1 & 292 \\
\hline S201412190939A & 2014-12-19 & 09:39:41 & 43.60 & 11.25 & 10.0 & -0.810 & 1.590 & -0.780 & -0.280 & -0.230 & -2.120 & 2.840 & 2 & 210 & -0.720 & 75 & 113 \\
\hline S201412191036A & 2014-12-19 & 10:36:32 & 43.66 & 11.13 & 10.0 & -2.240 & 2.420 & -0.190 & -1.460 & -0.470 & -3.390 & 4.880 & 8 & 213 & -1.230 & 46 & 115 \\
\hline S201412241140A & 2014-12-24 & 11:40:12 & 41.65 & 14.91 & 20.0 & 2.240 & -2.320 & 0.080 & 0.410 & -1.320 & -1.780 & 3.130 & 57 & 66 & 0.150 & 33 & 239 \\
\hline S201412282143A & 2014-12-28 & 21:43:38 & 39.29 & 16.36 & 11.0 & -0.790 & -0.070 & 0.860 & 0.410 & -0.060 & 0.160 & 0.880 & 0 & 279 & 0.100 & 25 & 10 \\
\hline C201412292034A & 2014-12-29 & 20:34:13 & 41.66 & 19.23 & 10.0 & 1.470 & -0.440 & -1.030 & 0.680 & -0.520 & 0.910 & 1.710 & 74 & 20 & 0.190 & 8 & 140 \\
\hline S201501300045A & 2015-01-30 & 00:45:49 & 46.41 & 13.14 & 10.0 & -0.060 & -0.750 & 0.810 & 0.470 & 1.430 & -0.070 & 1.890 & 37 & 276 & -0.490 & 23 & 25 \\
\hline S201502060852A & 2015-02-06 & 08:52:26 & 38.74 & 15.22 & 274.0 & -1.750 & 0.740 & 1.020 & -0.830 & -0.100 & 1.050 & 2.050 & 10 & 134 & -0.020 & 16 & 226 \\
\hline S201502280316A & 2015-02-28 & 03:16:17 & 41.98 & 13.53 & 17.0 & -4.350 & 2.170 & 2.190 & -1.320 & 0.170 & -2.490 & 4.790 & 7 & 224 & -0.160 & 10 & 133 \\
\hline S201502280824A & 2015-02-28 & 08:24:24 & 43.64 & 18.42 & 10.0 & -1.150 & 0.340 & 0.810 & 0.670 & -0.190 & 1.210 & 1.840 & 6 & 311 & -0.230 & 34 & 45 \\
\hline S201503040000A & 2015-03-04 & 00:00:04 & 43.60 & 11.18 & 10.0 & -0.170 & 0.080 & 0.090 & 0.020 & -1.070 & -0.790 & 1.350 & 31 & 58 & -0.030 & 36 & 173 \\
\hline S201503082047A & 2015-03-08 & 20:47:26 & 44.07 & 19.88 & 10.0 & -0.580 & -0.720 & 1.290 & -1.610 & -0.490 & 7.630 & 8.210 & 9 & 132 & -0.690 & 78 & 272 \\
\hline S201504010733A & 2015-04-01 & 07:33:53 & 44.33 & 16.41 & 10.0 & 0.250 & -2.220 & 1.970 & -1.610 & -1.140 & -0.550 & 2.550 & 28 & 94 & 0.660 & 48 & 221 \\
\hline S201504120005A & 2015-04-12 & 00:05:31 & 43.76 & 18.56 & 2.0 & -0.080 & -1.880 & 1.970 & -0.550 & -0.430 & 0.890 & 2.290 & 13 & 104 & -0.110 & 72 & 239 \\
\hline S201504151657A & 2015-04-15 & 16:57:02 & 38.88 & 15.49 & 256.0 & -1.620 & -1.340 & 2.960 & -1.810 & -1.930 & -0.080 & 3.730 & 22 & 97 & -0.090 & 32 & 202 \\
\hline S201505090822A & 2015-05-09 & 08:22:42 & 38.58 & 15.38 & 212.0 & -0.800 & 0.500 & 0.310 & -0.930 & -0.200 & -0.040 & 0.990 & 28 & 174 & 0.320 & 4 & 82 \\
\hline S201505110826A & 2015-05-11 & 08:26:32 & 37.33 & 16.80 & 40.0 & 2.970 & -1.530 & -1.440 & 2.480 & -0.810 & -6.610 & 6.640 & 33 & 42 & 1.590 & 56 & 236 \\
\hline S201505211531A & 2015-05-21 & 15:31:18 & 37.68 & 19.85 & 15.0 & -0.440 & 3.470 & -3.020 & -1.440 & 0.160 & 2.180 & 4.500 & 15 & 164 & -0.700 & 71 & 308 \\
\hline S201505220631A & 2015-05-22 & $06: 31: 16$ & 37.67 & 19.84 & 10.0 & 0.190 & 1.830 & -2.020 & -0.390 & 0.130 & 1.500 & 2.390 & 8 & 161 & 0.160 & 80 & 309 \\
\hline S201505291307A & 2015-05-29 & 13:07:57 & 42.92 & 14.30 & 10.0 & 1.000 & -0.330 & -0.670 & 0.340 & 0.240 & 0.270 & 1.130 & 72 & 327 & -0.310 & 18 & 153 \\
\hline S201507221257A & $2015-07-22$ & 12:57:44 & 44.16 & 10.89 & 10.0 & -0.280 & 0.330 & -0.050 & -0.610 & -0.130 & -0.780 & 1.070 & 18 & 213 & -0.060 & 48 & 102 \\
\hline S201508020658A & 2015-08-02 & 06:58:06 & 39.34 & 15.69 & 240.0 & -0.860 & 2.100 & -1.250 & -0.880 & -0.410 & -1.500 & 2.800 & 11 & 199 & -0.600 & 59 & 9 \\
\hline S201508030727A & 2015-08-03 & 07:27:48 & 39.16 & 16.53 & 26.0 & -2.890 & 1.540 & 1.350 & -0.070 & -1.090 & 1.790 & 3.340 & 8 & 135 & -0.160 & 14 & 43 \\
\hline S201508082246A & 2015-08-08 & 22:46:24 & 38.55 & 14.26 & 14.0 & -0.180 & 0.500 & -0.320 & -0.110 & -0.190 & -1.300 & 1.460 & 1 & 36 & -0.140 & 79 & 131 \\
\hline S201508292025A & $2015-08-29$ & 20:25:14 & 38.39 & 12.14 & 10.0 & 3.000 & -3.060 & 0.060 & -2.990 & -0.100 & -1.390 & 4.270 & 66 & 195 & 0.390 & 12 & 78 \\
\hline S201508291847A & 2015-08-29 & 18:47:04 & 46.31 & 13.58 & 7.0 & 0.440 & -1.220 & 0.780 & 0.110 & 0.360 & 0.230 & 1.040 & 32 & 278 & 0.210 & 58 & \\
\hline S201509130104A & 2015-09-13 & 01:04:35 & 43.62 & 11.16 & 5.0 & -0.420 & 0.320 & 0.100 & -0.740 & 0.160 & -1.070 & 1.520 & 19 & 219 & -0.380 & 49 & 105 \\
\hline S201510141020A & 2015-10-14 & 10:20:53 & 38.95 & 14.63 & 287.0 & -2.210 & -1.780 & 3.990 & -1.660 & -1.440 & -0.780 & 4.340 & 11 & 86 & -0.350 & 39 & 185 \\
\hline S201510160016A & $2015-10-16$ & 00:16:24 & 39.33 & 15.65 & 244.0 & -2.290 & 1.070 & 1.220 & -3.830 & -1.130 & -1.890 & 3.980 & 25 & 205 & 1.420 & 27 & 101 \\
\hline S201511010752A & 2015-11-01 & 07:52:32 & 45.87 & 15.56 & 10.0 & 4.390 & -5.090 & 0.700 & -2.990 & 1.540 & 1.080 & 5.550 & 70 & 226 & 0.710 & 10 & 106 \\
\hline S201511060403A & 15-11-06 & :03:05 & & & 10.0 & -1.070 & 0.210 & 0.870 & 0.100 & 0.790 & 120 & 1.160 & 19 & 265 & 0.210 & & 357 \\
\hline S201512061624A & 2015-12-06 & 16:24:40 & 42.34 & 15.08 & 10.0 & 0.760 & -0.160 & -0.600 & 0.330 & -0.410 & -0.340 & 1.020 & 63 & 42 & -0.200 & 25 & 199 \\
\hline S201512060528A & $2015-12-06$ & 05:28:15 & 42.39 & 15.12 & 10.0 & 3.930 & -1.050 & -2.890 & 1.460 & -1.830 & -1.850 & 4.990 & 65 & 43 & -0.880 & 24 & 206 \\
\hline S201512060322A & 2015-12-06 & $03: 22: 49$ & 42.65 & 15.17 & 10.0 & 4.020 & -0.640 & -3.380 & -0.620 & 1.030 & -1.710 & 4.310 & 76 & 223 & -0.060 & 13 & ${ }^{23}$ \\
\hline & 2015-12-20 & t4.03 & 38.3 & 13.58 & 10.0 & 3.250 & -1.230 & 2.020 & -4.000 & -2.090 & -1.360 & 5.770 & 61 & 162 & -0.570 & 10 & 54 \\
\hline S201512291415A & 2015-12-29 & 14:15:53 & 37.42 & 19.89 & 10.0 & -0.260 & 0.950 & -0.690 & 0.010 & 0.840 & 0.880 & 1.420 & 13 & 332 & 0.130 & 55 & 223 \\
\hline
\end{tabular}

